\title{
APPLICATION OF INTERACTIVE EVOLUTIONAL COMPUTING TO CORE DESIGN
}

\author{
Yoko Kobayashi \\ TEPCO SYSTEMS CORPORATION \\ 2-37-28 Eitai Koto-ku Tokyo 135-0034, Japan \\ kobayashi-youko@tepsys.co.jp
}

\begin{abstract}
This paper presents a new approach to the use interactive evolutional computing (IEC) for the automatic core design of a boiling water reactor (BWR). In particular, the approach combines the characteristics of both heuristic search and interactive operation. A heuristic search is performed by a population of chromosomes (or agents) in a search space and an interactive operation is performed by a skilled engineer. These results are competitive with our previous technique. The effectiveness of this approach is demonstrated by the reduction in time and number of iterations it takes to find an optimal loading pattern in the core design of a real BWR. Copyright (C) 2005 IFAC
\end{abstract}

Keywords: Interactive Evolutionary Computing, Optimization, Multi-agents Algorithm, Core design

\section{INTRODUCTION}

The broader definition of interactive evolutionary computation (IEC) is "the technology that evolutional computing (EC) optimizations the target systems having an interactive human-machine interface." However, almost all previous researches of IEC based on the narrow definition, in which fitness function is replaced with a human user, and the EC searches for the global optimum solution in a feature parameter space according to the psychological distance. EC is a biologically inspired general computational concept such as genetic algorithms (GA), evolutional strategy (ES), genetic programming (GP), and evolutionary programming (EP). The IEC application fields include graphic arts (Sims, 1991), speech processing (Watanabe, 1995), synthesis (Biles, 1994), and industrial design (Parmee, 1993). Recently, the applications of IEC have been extended to become practicable for the solution of engineering problems.

On the other hand, boiling water reactor (BWR) is one of the commercial nuclear power plants. The task of deciding the optimal locations of a number of fuel assemblies while considering the safety and economy of the reactor is called the core design. The combination of these optimal locations is called the loading pattern (LP). To control the core reactivity while operating a BWR, several control rods (CR) are inserted. The optimal axial locations of these CRs are called the control rod pattern (CRP). It is necessary to optimize both the LP and the CRP at the same time in the actual core design of a BWR.

For the last several years, we have been applying genetic algorithms (GA) and multi-agent algorithms (MAA) without transcendental information to optimize the core design of BWR. We were able to achieve the automatic optimization of the complex core design of a BWR by these approaches. This has great significance in labor saving and the maintenance of constant performance quality. However, it is very wasteful not to use the expertise of skilled engineers. If the above automatic optimization algorithm has the same level of performance as that of the skilled engineer, further improvement of the performance can be expected by using both the engineer and IEC in parallel.

In this paper, we propose an original technique using an IEC for the automatic core design of a BWR. The goal of this study is to accelerate the progress to a solution and use the ability of skilled engineers effectively. In our new technique, the skilled engineer's technical expertise is included in 
the search process by using IEC while evaluating the fitness function of current generation.

\section{FORMULATION OF BWR CORE DESIGN}

As I explained in the previous section, the core design of a BWR includes the optimization of both LP and CRP. The core design is one of the most important tasks in core fuel management and is a very complex optimization problem. Fig. 1 shows the procedure of BWR core design. This procedure is summarized as follows:

Step1: Set the initial LP and CRP.

Step2: Evaluate the core performance by three-dimensional reactor physics code.

Step3: Check whether all constraint conditions are satisfied.

Step4: If all constraint conditions are satisfied, the procedure is finished.

Step5: Otherwise, shuffling of the LP is performed.

Step6: A CRP suitable to each LP is sought.

Step7: Go to Step2

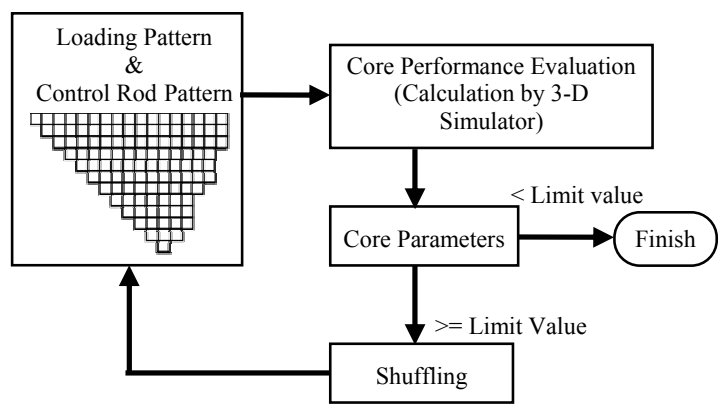

Fig.1 Procedure of BWR Core Design

We formulate this optimization problem in this section. Here, consider an octant core as shown in Fig.2. When all fuel is numbered in the order of reactivity, the fuel assembly placed in loading position 1 is defined as $\mathrm{xl}$ and this list is arranged as $x=\left(x_{1}, \cdots, x_{l}, \cdots, x_{L}\right),\left(L=1, \cdots, N_{F}\right)$, where $x_{l} \neq x_{l^{\prime}}, \quad l \neq l^{\prime}, \quad x_{l}, x_{l^{\prime}} \in\{1, \cdots, L\} . \quad N_{F}$ is the number of fuel assemblies. Then, if the axial position of the CR $n\left(n=1, \cdots, N_{c}\right)$ at each burn-up step $t$ is defined as $y_{n}(t)$, the list is expressed as $y(t)=\left(y_{1}(t), \cdots, y_{n}(t), \cdots, y_{N c}(t)\right)$ as shown in Fig.3. There are $N t$ burn-up steps including both BOC (beginning of cycle) and EOC (end of cycle), and defined as $t=\left(1, \cdots, N_{t}\right)$. Evaluation of this list $\boldsymbol{x}$ is performed with a three-dimensional diffusion code coupled with neutronic and thermal hydraulic models. We define the following parameters as the constraint conditions for an advanced BWR, (ABWR) core design:

$e_{f}(t), \overline{e_{f}}(t): k_{\text {eff }}$, the target value of $k_{\text {eff }}$,

$r(t), \bar{r}(t)$ : rerative nodal power, the upper limit value of relative nodal power, flcpr $(t), \overline{f l c p r}(t)$ : FLCPR (fraction of limiting critical power ratio), the upper limit value of FLCPR,

$f l p d(t), \overline{f l p d}(t)$ : FLPD (fraction of limiting power density), the upper limit value of FLPD,

$s d m(t), \overline{s d m}(t)$ : SDM (cold shutdown margin), the limit value of SDM.

As constraints of operation conditions, the following items are considered:

$\overline{\text { flow }}_{\text {EOC }}^{\text {upper }}$ : the upper limit value of core flow rate at $\mathrm{EOC}$,

flow $(t)$ : the core flow rate at each burn-up step $t$ (Constant rate).

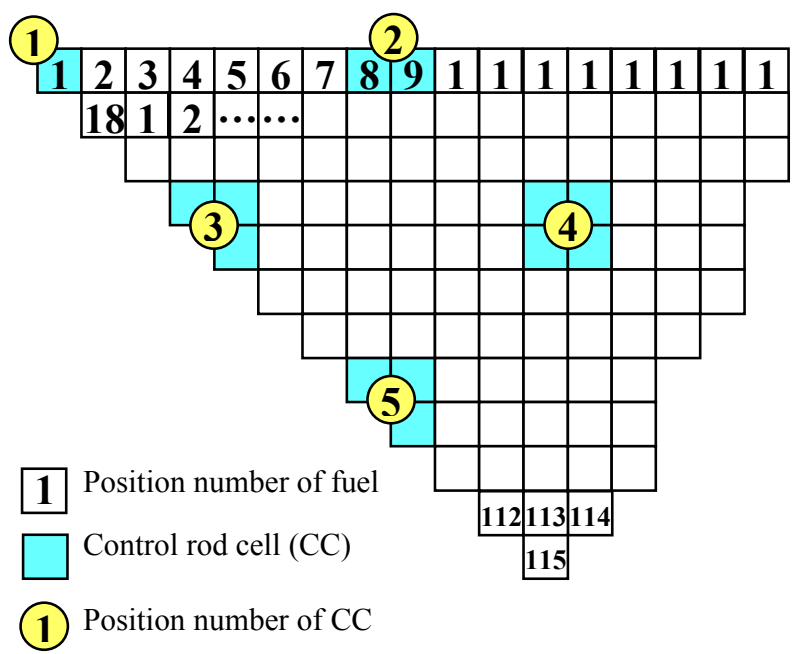

Fig. 2 Position numbers of fuel and locations of control rods ( example of 5CC)

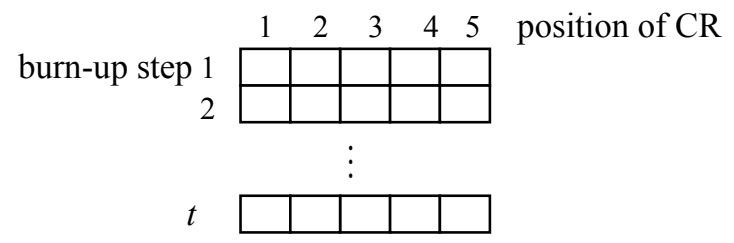

Fig. 3 Sample coding of chromosome in the second stage

In the master-servant relationship between LP and CRP, it is important that the set of CRP $y$ searched for later agrees with the target value of $\overline{e_{f}}(t)$ for the set of LP searched for first. Such a pattern $\boldsymbol{x}$ is called a controllable LP, and the set is expressed as Eq. (1) using tolerance $\varepsilon$ :

$X=\left\{\boldsymbol{x}|| e_{f}(t)-\overline{e_{f}}(t) \mid<\varepsilon, t=1, \cdots, N_{t} \quad\right.$ for $\exists \boldsymbol{y}(\bullet)$,

where $\boldsymbol{y}(\bullet)=\left(y(1), \cdots, y\left(N_{t}\right)\right)$. As the control rod position, constrained set $\mathrm{Y}$ concerning $\boldsymbol{y}(\bullet)$ is given by: 
$Y=\left\{y(\bullet) \mid y_{n}(t) \in\left\{\operatorname{pos}_{\text {in }}, \cdots, \operatorname{pos}_{\text {out }}, \operatorname{pos}_{\text {all }}\right\}, t=1, \cdots, N_{t}\right\}$

where $\operatorname{pos}_{\text {in }}$, pos $_{\text {out }}$, and pos all are the limitation of insertion of control rods, the limitation of drawing, and the all rods out. From this, the two-stage optimization problem is formulated as:

$$
\begin{aligned}
\min _{\boldsymbol{x}} d_{1} g_{1}(\boldsymbol{x}, \overline{\boldsymbol{y}}(\bullet)) & +d_{2} g_{2}(\boldsymbol{x}, \overline{\boldsymbol{y}}(\bullet))+d_{3} g_{3}(\boldsymbol{x}, \overline{\boldsymbol{y}}(\bullet)) \\
& +d_{4} g_{4}(\boldsymbol{x}, \overline{\boldsymbol{y}}(\bullet))+d_{5} P(\boldsymbol{x}, \overline{\boldsymbol{y}}(\bullet)),
\end{aligned}
$$

subject to $\boldsymbol{x} \in \boldsymbol{X}$,

$$
g_{0}(\boldsymbol{x}, \overline{\boldsymbol{y}}(\bullet))<\varepsilon,
$$

where $\overline{\boldsymbol{y}}(\bullet)=\arg \min _{\boldsymbol{y}(\bullet) \in Y} g_{0}(\boldsymbol{x}, \boldsymbol{y}(\bullet))$,

$\left.g_{1}(\boldsymbol{x}, \overline{\boldsymbol{y}}(\bullet))=\max _{t}\left\{\max _{(m, l)}\left\{\max \left\{r_{l m}(t)\right)-\bar{r}(t), 0\right\}\right\}\right\}$,

$g_{2}(\boldsymbol{x}, \overline{\boldsymbol{y}}(\bullet))=\max _{t}\left\{\max _{l}\left\{\max \left\{f l c p r_{l}((t))-\overline{f l c p r}(t), 0\right\}\right\}\right\}$,

$g_{3}(\boldsymbol{x}, \overline{\boldsymbol{y}}(\bullet))=\max _{t}\left\{\max _{(m, l)}\left\{\max \left\{f l p d_{l m}((t))-\overline{f l p d}(t), 0\right\}\right\}\right\}$,

$g_{4}(\boldsymbol{x}, \overline{\boldsymbol{y}}(\bullet))=\sum_{t=2}^{N_{t}} \sum_{k=1}^{N_{c}}\left\{y_{k}(t)-y_{k}(t-1)\right\}$,

$P(\boldsymbol{x}, \overline{\boldsymbol{y}}(\bullet))=\max \left\{\operatorname{flow}\left(\boldsymbol{x}, \boldsymbol{y}\left(N_{t}\right)\right)-\overline{f l o w_{E O C}^{u p p e r}}, 0\right\}$,

$x_{l} \neq x_{l}, l \neq l^{\prime}, \quad x=\left(x_{1}, \cdots, x_{l}, \cdots, x_{L}\right), x_{l}, x_{l^{\prime}} \in\{1, \cdots, L\}$,

$y_{n}(t) \in\left\{\operatorname{pos}_{\text {in }}, \cdots\right.$, pos $_{\text {out }}$, pos $\left._{\text {all }}\right\}$.

$\boldsymbol{y}(t)=\left(y_{1}(t), \cdots, y_{n}(t), \cdots, y_{N_{c}}(t)\right), \quad t \in\left\{t_{1}, \cdots, t_{N_{t}}\right\}$,

The values of $d_{1}$ to $d_{5}$ in Eq. (3) are determined by some trial-and-error based on the empirical rule obtained by the reload core design of a real plant. In a core with a severe cold shutdown margine (SDM), it is necessary to add the term of SDM to the objective function of Eq.(3). This is a two-stage combinatorial optimization problem with a nonlinear objective function and nonlinear constraints.

\section{OUR PREVIOUS APPROACHES}

In this section, we describe our previous approaches for the automatic core design of a BWR which we formulate in section 2 . The automatic core design of a BWR is very complex optimization problem. In order to solve such complex problem, powerful and efficient optimization technique is desired. First, we developed an Improved Genetic Algorithm (GA) (Kobayashi, 2000). The main features of this improved GA are summarized as follows:

- Generation changes are performed by the comparison of the fitness function between the previous generation and the current generation,

- Performance of the GA is improved by the execution of the deterministic operators (both crossover rate and mutation rate are 1.0),

- Convergence efficiency is raised by the adoption of an original elite strategy that utilizes the fact that the LP problem is a two-objective problem, and

- Convergence efficiency is raised further by self-reproduction done every several generation.

The rough optimal number of fresh fuel assemblies can be estimated using this algorithm.

Next, we developed the integrative optimization technique using two-stage GA (Kobayashi, 2002). In the first stage of the two-stage GA, optimization of the LP is performed using improved GA. In the second stage, the optimal CRP corresponding to each LP is sought using GA with two-dimensional coding of chromosome. Further, a forceful heuristic if-then rule is introduced in the second stage to improve the convergence ability. This two-stage GA provides both good global and local searching ability. It achieves the automatic core design of a BWR within a reasonable computation. In addition, this technique can respond also to changes of design conditions, such as the change of a target value, promptly. After that, we developed the multi-agents algorithm (MAA) (Kobayashi, 2004) to improve the optimization ability. In the MAA, the optimal solution is obtained by information exchange using the coupling relations among multiple agents in the agent space. We applied this MAA to the first stage of the two-stage optimization technique described above.

\section{THE INCLUSION OF IEC}

Many ideas on IEC have been proposed in the control field. However, in the conventional research on IEC, either IEC was performed alone or IEC plus non-IEC were performed alternately. The IEC proposed in this paper uses a new concept in which the automatic optimization of the core design is performed using two-stage GA and IEC at the same time. The calculation time of one generation requires more than ten minutes for the automatic optimization of the core design of a BWR. Therefore, this time period is used, and the skilled engineer's expertise is used in the automatic optimization using IEC. The goal of this study is to accelerate the progress to a solution and to use the ability of skilled engineers effectively.

The basic procedure for including IEC is briefly explained as follows. Two maps are displayed on the screen of the computer. One is the LP of the best individual in the previous generation, and the other is a BOC exposure map corresponding to this LP. Three locations where the evaluation indexes (e.g., FLCPR, FLPD) are critical are indicated on the LP as shown in Fig.4. The skilled engineer makes the best use of his/her experience to shuffle the fuel assemblies on the screen from these two maps. In the 
example shown in Fig.4, two fuel assemblies are exchanged. The LP in which this shuffling is performed is replaced with the LP of the worst individual in the current generation, and the calculations of the GA (or MAA) of the next generation are continued. The inclusion of such IEC in the process is continued for several generations. Fig.5 illustrates the flow of these procedures.

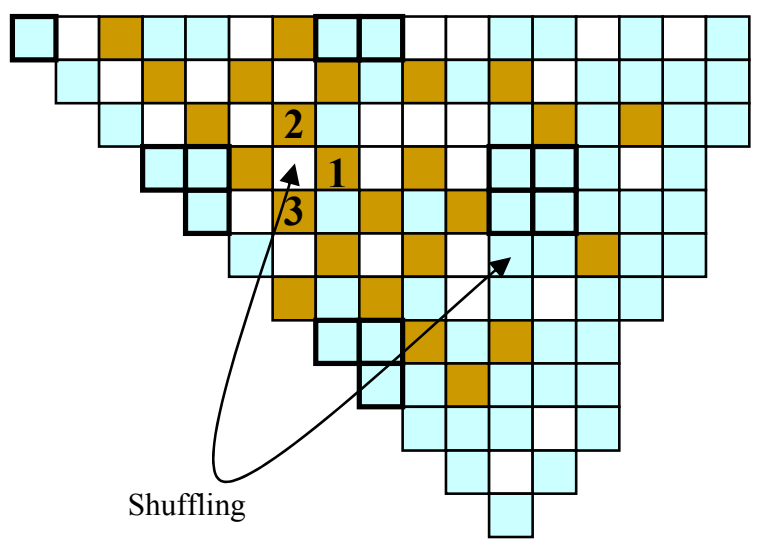

Fresh Fuel Assembly

1-Cycle Burned Fuel Assembly

Low-reactivity Fuel Assembly

Fig. 4 Example of shuffling using IEC

\section{SIMULATION RESULT}

In order to verify the effectiveness of IEC, test cases based on some rules were analyzed. The target reactor was an ABWR. The results concern how to determine the shuffling rules. The shuffling rules can be classified into three categories:

- The number of shuffles performed in one interaction,

- The number of generations that are shuffled,

- The rule of shuffling.

Let us consider the following test cases with IEC:

Case 1: Only one shuffle is performed by IEC in each generation, and this is done during the first 10 generations,

Case2: Two shuffles are performed by IEC in each generation, and this is done during the first 10 generations,

Case3: Only one shuffle is performed by IEC in each generation, and this is done during the first 15 generations,

Case4: Only one shuffle is performed by IEC in each generation, and this is done during the first 10 generations and the shuffling is limited to only one-cycle burned fuel assemblies.

For cases 1-4, the best shuffle is determined by an engineer. Special limitations on the shuffling are not created except for constraints of optimization.

Table 1 shows the parameter of LP optimization. $\bar{s}$ is upper generation numbers of LP, $\bar{t}$ is upper generation numbers of CRP, and $S c$ is interval generations of self-reproduction. We used 30 as the agent number $M$. Table 2 shows the parameters of CRP optimization. Table 3 shows target values in the objective functions. The results of case 1 were averaged over 10 runs and are shown in Fig. 6. The optimal LP was obtained at the 27th generation when IEC was performed until the 10th generation and one shuffle was executed in each generation. Better convergence ability than two-stage MAA, where the optimal LP was obtained at the 35th generation, was accomplished. When IEC was performed until the 10th generation and two shuffles were executed in each generation, convergence ability is almost the same as the case in which one shuffle per generation is performed. A comparison of cases 1-4 is shown in Table 4. Longer shuffling during more generations is effective. However, using too many generations has little advantage. Since the proposed IEC is a technique to search for the possibility of the creation of a good individual by experienced technology, it is effective for the early stage of the search.

Table 1 Parameters of LP optimization in the first stage

\begin{tabular}{cccc}
\hline$M$ & $\bar{s}$ & $\bar{t}$ & $S c$ \\
\hline 30 & 100 & 2 & 10 \\
\hline
\end{tabular}

Table 2 Parameters of CRP optimization in the second stage

\begin{tabular}{ccccc}
\hline Nc & Nt & Pos $_{\text {in }}$ & Pos $_{\text {out }}$ & Pos $_{\text {all }}$ \\
\hline 5 & 6 & 60 & 100 & 200 \\
\hline
\end{tabular}

Table 3 Target values

\begin{tabular}{|c|c|c|c|c|c|}
\hline$\overline{\overline{e_{f}}}\left(t_{1}\right)$ & $\overline{\overline{e_{f}}}\left(t_{2}\right)$ & $\overline{\overline{e_{f}}}\left(t_{3}\right)$ & $\overline{\overline{e_{f}}}\left(t_{4}\right)$ & $\overline{\overline{e_{f}}}\left(t_{5}\right)$ & $\overline{\overline{e_{f}}}\left(t_{6}\right)$ \\
\hline 0.9980 & 0.9974 & 0.9968 & 0.9963 & 0.9962 & 0.9986 \\
\hline $\bar{r}(t)$ & $\overline{f l c p r}(t)$ & $\overline{f l p d}(t)$ & $\overline{\text { flow }_{E O C}^{\text {upper }}}$ & $\varepsilon_{L P}$ & \\
\hline 1.70 & 0.95 & 0.95 & $111 \%$ & 0.0003 & \\
\hline
\end{tabular}




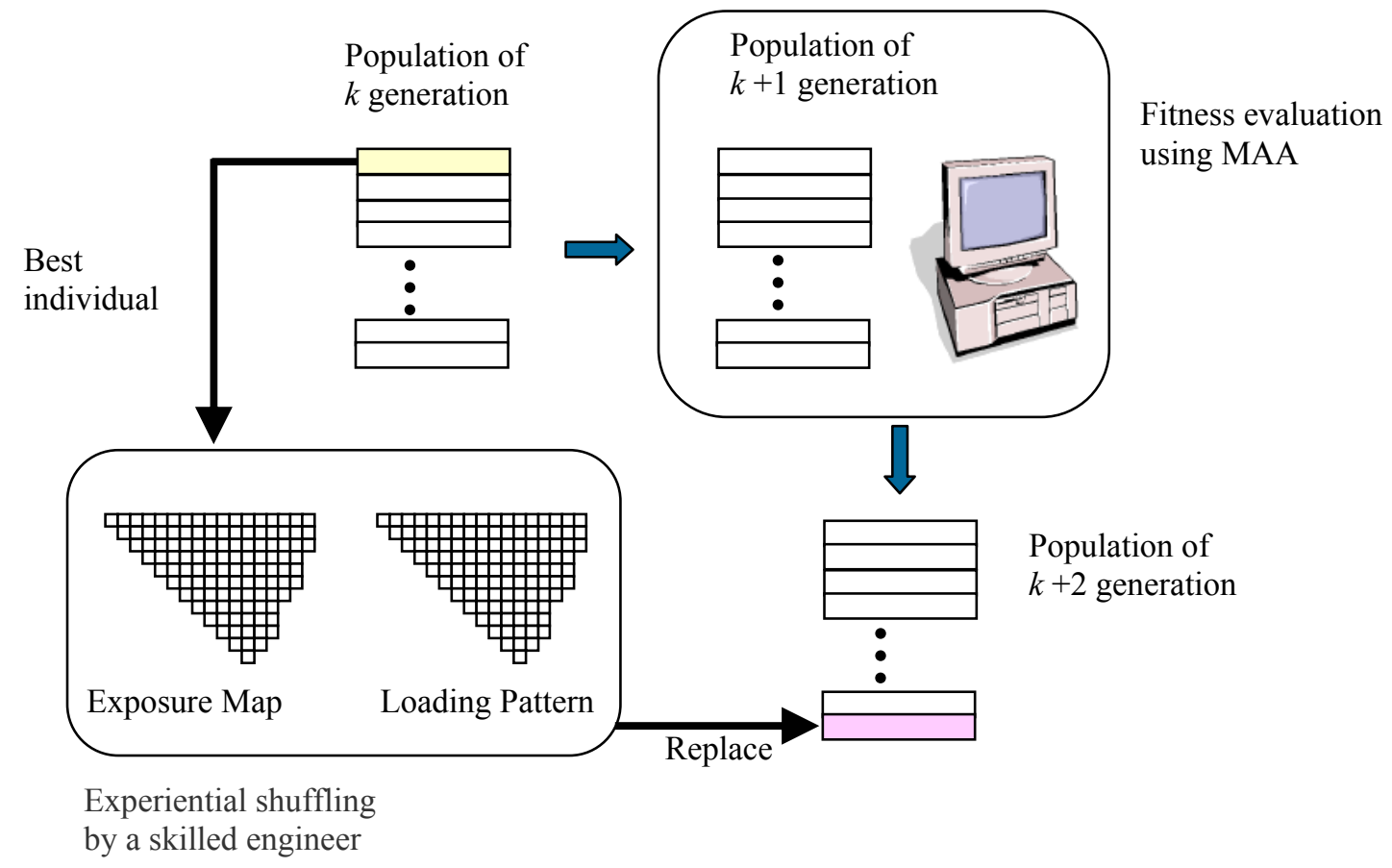

Fig. 5 Procedure of IEC \& non-IEC

MAA is a system in which multiple agents exchange information by coupling structures and coupling operations suitable for the assigned problem, and by acting autonomously. On the other hand, IEC understands the whole process of learning during the optimization, and is considered to be a system in which knowledge is included deterministically.

Table 4 Numbers of generations for optimum core design

\begin{tabular}{|c|c|c|c|c|c|}
\hline & \multirow{2}{*}{$\begin{array}{c}\text { Two-stage } \\
\text { MAA }\end{array}$} & \multicolumn{4}{|c|}{ IEC + MAA } \\
\hline & & Case 1 & Case 2 & Case 3 & Case 4 \\
\hline $\begin{array}{l}\text { Generation } \\
\text { Numbers for } \\
\text { Optimum } \\
\text { pattern }\end{array}$ & & & & & \\
\hline Average & 35 & 27 & 25 & 26 & 30 \\
\hline Worst & 63 & 53 & 51 & 53 & 58 \\
\hline Best & 13 & 11 & 11 & 11 & 12 \\
\hline
\end{tabular}

\section{CONCLUSION}

In this paper, we proposed an optimization technique in which a skilled engineer's technical knowledge is included in the use of IEC in the automatic core design of a BWR using MAA. The performance of IEC was analyzed. The convergence performance was improved by more than $30 \%$ compared with the conventional MAA alone. Moreover, the skilled engineer's technical knowledge can be effectively used by this method. The technique proposed in this paper is an innovative optimization using an interactive operation and has a special meaning as a regulatory function. Of course, the efficiency of IEC depends on the method of inserting IEC (the number of shuffles, the number of generations in which shuffling is executed, etc.)
Various methods for the inclusion of IEC are available in addition to using the method proposed in this paper. If the mechanism of the inclusion of IEC is systematized, a more powerful algorithm will be created.

\section{REFERENCES}

Biles, J.A., (1994). Computational Organization Theoty, Lawrence Erlbaum Associates.

Kobayashi, Y., Aiyoshi, E., (2002). Optimization of Boiling Water Reactor Loading Pattern Using Genetic Algorithm, Nuclear Science and Engineering, Vol.142, pp.119-139.

Kobayashi, Y., Aiyoshi, E., (2000). Optimization of Boiling Water Reactor Loading Pattern Using Improved Genetic Algorithm, Proceeding of American Nuclear Society International Topical Meeting on Nuclear Plant Instrumentation Control and Human-Machine Interface Technologies, Washington DC, November, pp.134-156.

Kobayashi, Y., Aiyoshi, E., (2004). Automatic Core Design Using Reinforcement Learning, American Control Conference 2004, Boston

Parmee, I.C., (1993). The concrete arch dam: an evolutionary model of the design process, Int'l Conference on Artificial Neural Nets and Genetic Algorithms, Innsbruck, Austria, pp.14-16.

Sims, K., (1991). Artificial evolution for computer graphics, Computer Graphics, Vol.25, 4, pp.319-328.

Watanabe, T., Takagi, H., (1995). Recovering system on the distorted speech using interactive genetic algorithms, IEEE Int'l Conference on Systems Man and Cybernetics, Vol.1, pp.684-689. 